\author{
Marquette University \\ e-Publications@Marquette
}

Electrical and Computer Engineering Faculty Research and Publications

Electrical and Computer Engineering,

Department of

\title{
Improving Gold/Gold Microcontact Performance and Reliability Under Low-Frequency AC Through Circuit Loading
}

T. Laurvick

Air Force Institute of Technology

Ronald A. Coutu Jr.

Marquette University, ronald.coutu@marquette.edu

Follow this and additional works at: https://epublications.marquette.edu/electric_fac

Part of the Computer Engineering Commons, and the Electrical and Computer Engineering Commons

\section{Recommended Citation}

Laurvick, T. and Coutu, Ronald A. Jr., "Improving Gold/Gold Microcontact Performance and Reliability Under Low-Frequency AC Through Circuit Loading" (2016). Electrical and Computer Engineering Faculty Research and Publications. 320.

https://epublications.marquette.edu/electric_fac/320 
Marquette University

\section{e-Publications@Marquette}

\section{Electrical and Computer Engineering Faculty Research and Publications/College of Engineering}

This paper is NOT THE PUBLISHED VERSION; but the author's final, peer-reviewed manuscript. The published version may be accessed by following the link in the citation below.

IEEE Transactions on Components, Packaging and Manufacturing Technology, Vol. 7, No. 3 (September, 2016). DOI. This article is (C) Institute of Electrical and Electronic Engineers (IEEE) and permission has been granted for this version to appear in e-Publications@Marquette. Institute of Electrical and Electronic Engineers (IEEE) does not grant permission for this article to be further copied/distributed or hosted elsewhere without the express permission from Institute of Electrical and Electronic Engineers (IEEE).

\section{Contents}

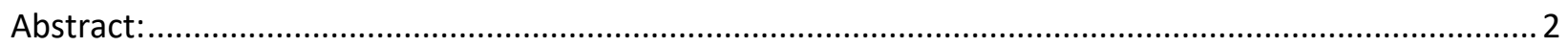

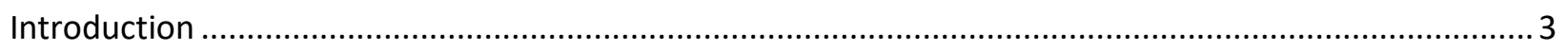

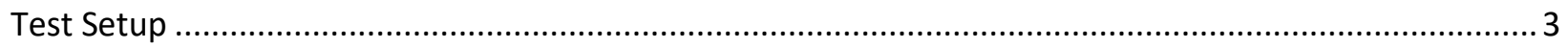

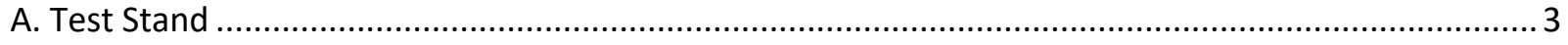

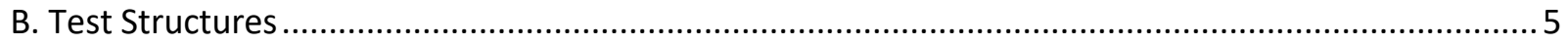

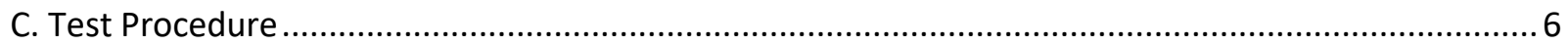

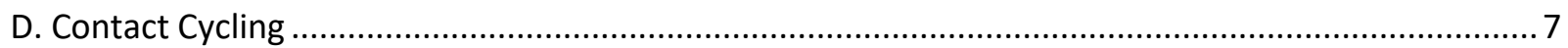

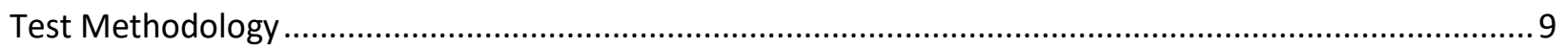

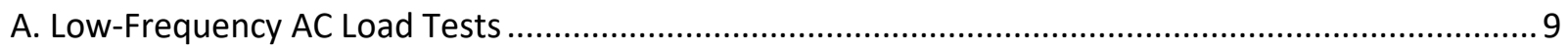

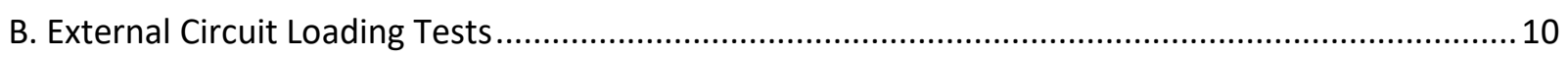

C. Low-Frequency AC Tests With Circuit Loading Mitigations .............................................. 12

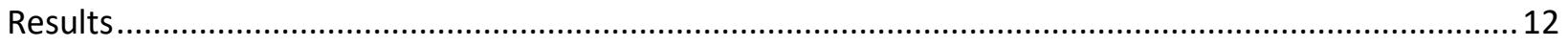

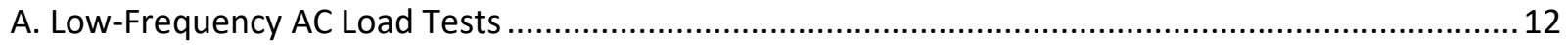

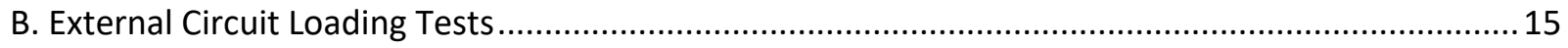

C. Low-Frequency AC Tests With Circuit Loading Mitigations .............................................. 19 
Conclusion.

Acknowledgement

\title{
Improving Gold/Gold Microcontact Performance and Reliability Under Low- Frequency AC Through Circuit Loading
}

\author{
Tod V. Laurvick
}

Department of Electrical and Computer Engineering, Air Force Institute of Technology, Wright-Patterson $\mathrm{AFB}, \mathrm{OH}$

Ronald A. Coutu

Department of Electrical and Computer Engineering, Air Force Institute of Technology, Wright-Patterson $\mathrm{AFB}, \mathrm{OH}$

\section{Abstract:}

This paper investigates the performance and reliability of microcontacts under low-frequency and lowamplitude ac test conditions. Current microcontact theory is based on dc tests adapted to RF applications. To help better apply dc theory to RF applications, frequencies between $100 \mathrm{~Hz}$ to $100 \mathrm{kHz}$ were experimentally investigated. Microcontacts designed to conduct performance and reliability measurements were used, which in prior dc testing typically lasted for 100 million cycles or more. Under ac loads, at similar power levels, eight devices were tested under cold-switching conditions, and only one was still operational at 10 million cycles. The effect of external circuitry on dc loaded devices was also considered. The experimental data were presented for dc conditions, which demonstrated that both a parallel capacitance with a microcontact and a series inductance were highly detrimental. For all six tested devices, failure occurred typically in 100 thousand cycles or less. However, utilizing series resistive/capacitive circuits as well as parallel resistor/inductive resulted in improved performance, with only one device of the four tested failing prematurely, but those that lasted showed less variation in measure contact resistance throughout the lifetime of the device. Two devices were tested with passive contact protection using parallel and series resistances, and both devices lasted for the full test duration. Finally, the effects of applying circuit protection to microcontacts and repeating ac test conditions were investigated. Reliability and device lifetime were extended significantly $(9.1 \%$ success rate without protection was increased to $87 \%$ success rate). It was also observed in several instances that devices that failed showed subtle signs of variance during contact closure measurements in the 
range of 5-30 $\mu \mathrm{N}$, indicating a possible means for accurately predicting device failure. For these failed devices, notable physical damage was observed using a scanning electron microscope.

\section{Introduction}

Microswitches have been under investigation for years, and a great deal of research has occurred recently to address specific concerns in applying these devices to RF applications. ${ }^{1-2,3}$ The many benefits of using these microelectromechanical system (MEMS) devices are apparent, yet reliability remains an issue. ${ }^{4}$ Most of our most current theory is based on results obtain under dc conditions. Thus, application of this theory may not directly relate to RF application. Until performance and reliability are better understood, mitigation is difficult, and until we are able to apply mitigation, utilization on a large scale will be limited. To help break this barrier, we need an understanding of how to apply our dc-based predictions, starting with low-frequency ac applications. Then with this understanding, we can better predict the effects of increased frequency and understand device breakdown under these conditions. Thus, investigations such as this are crucial to understanding how best to mitigate these failures.

At the heart of this study are the observations that the polarity of a dc load applied to a microcontact can be critical to its long-term performance and that material transfer due to electromigration ${ }^{5}$ can be induced under low-frequency ac loads. During closure, asperities resulting from the roughness of the surface may induce high current densities. ${ }^{6}$ which then promote extremely localized joule heating and can change contact geometry, ${ }^{7}$ increasing contact resistance, which then promotes more heating. ${ }^{8}$ Typically, a combination of these two effects (high current densities ultimately leading to electromigration) has been observed repeatedly and attributed to contact failure. Under any ac conditions, devices are exposed to a constantly alternating polarity. However, if polarity is critical to microcontact reliability, then we are operating these devices in a manner where favorable and unfavorable polarities are constantly imposed. Understanding the effects of this mode of operation and effective mitigation techniques for this becomes critical, and investigating lower frequencies helps simplify the task.

Looking to prior efforts in mitigating these device failures, it has been demonstrated that external circuitry can have a significant effect under dc conditions. ${ }^{9}$ While the motivation for this protective circuitry has been to address hot-switching damage, testing the effectiveness of this sort of protection with low-frequency ac loads is the next task. Thus, the goal is to investigate not only how microcontacts behave during closure with an ac load, but also the effects of this polarity reversal during cold-switching in a closed microcontact, how performance and reliability are altered under these conditions, and whether these effects be can mitigated.

\section{Test Setup}

\section{A. Test Stand}

To better control experimental complexity and minimize the number of variables affecting the results, these experiments utilized microcontacts that were designed to be externally actuated. A test stand designed specifically to gather data on the contacting surfaces was used under controlled test conditions. This stand applied a known controllable contact force throughout all stages of the experiment, calculating contact resistance measurements from currents and voltages measured using an $\mathrm{NI}-4070$ FlexDMM module $\pm 110 \mathrm{n} \Omega$ of accuracy). The actuation position was controlled with a ThorLAB 
PAZO05 actuator, and the force was measured using a FemptoTools model FT-5270 sensor. MEMS microcontacts were fabricated for use with this test stand, allowing for several controlled tests to be conducted, each evaluating variables of interest.

The test stand designed to conduct this testing is shown in Fig. $1 .{ }^{10}$ It was composed of an enclosure in which dry nitrogen was used to minimize atmospheric contamination during testing. Three micromanipulators provide movement in the $x-, y-$, and $z$-axes, allowing alignment between the force sensor mechanism and each microcontact. The top inset of Fig. 1 shows a single reticle containing 16 test devices, all of which were externally wired to the same control rack, which monitored the force sensor and controlled the piezo actuator. The testing cycle then autonomously alternated between two repeated processes (measurement and wear), until either the desired number of test cycles was achieved or the device failed.
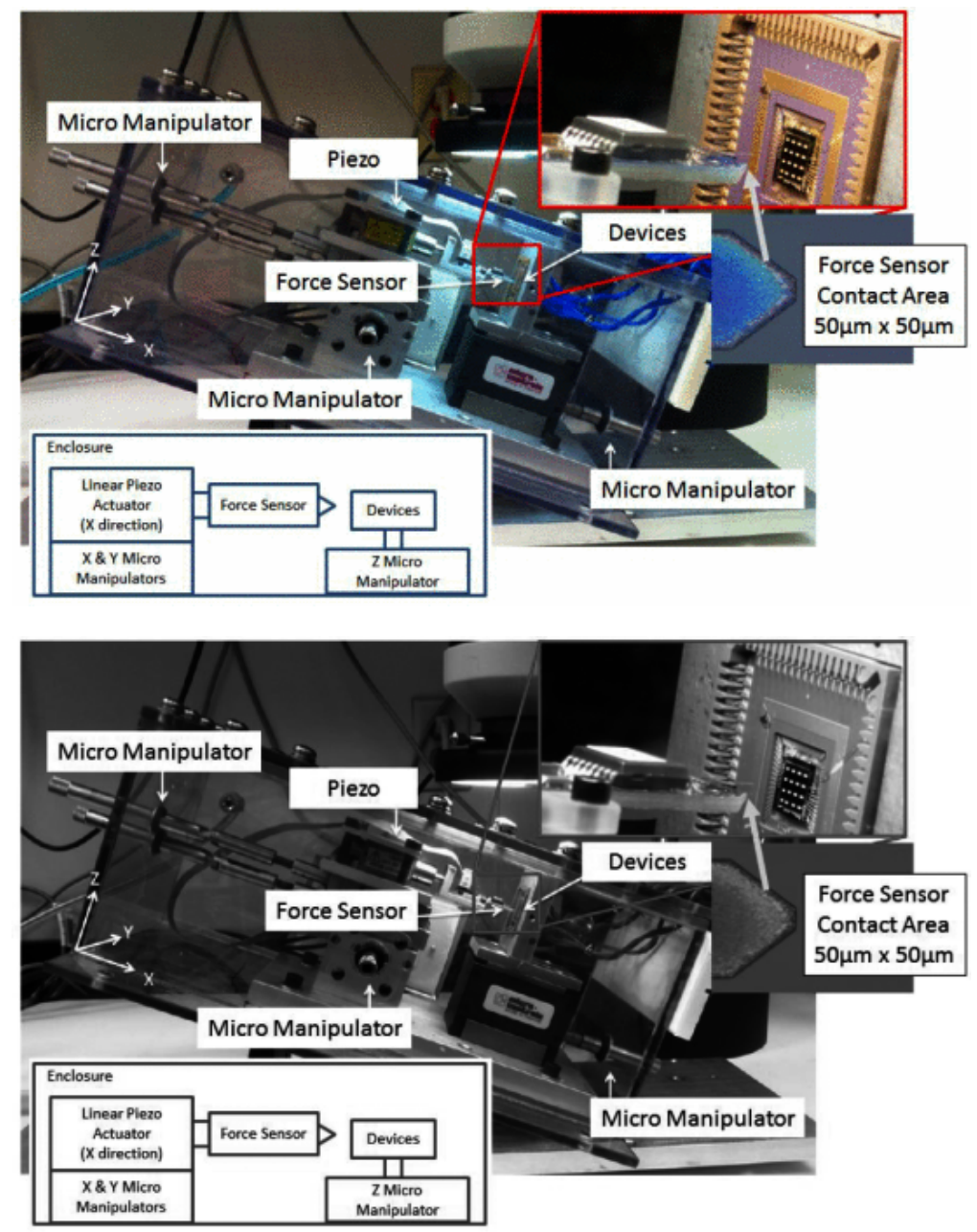

Fig. 1. Contact resistance test stand image and schematic utilizing a piezo-controlled force sensor which engages externally wired micro-contacts (top inset) and schematic representations of connecting subsystems (lower inset). 


\section{B. Test Structures}

An illustration of a test device is shown in Fig. 2. This design allows for the contact resistance measurement at any force applied to the beam, with simultaneous application of an electrical load upon the device to simulate operation. This was accomplished through the four connection points shown (required to conduct the Holm cross-bar measurement ${ }^{6}$ ), which connect to both sides of the fixed-fixed upper beam and both sides of lower thin-film contact. When aligning the force sensor, the tip was placed directly above the center of this upper beam to allow the piezo actuator to close the contact. The underside of this upper beam was fabricated with a hemispherical contact bump $8 \mu \mathrm{m}$ in radius protruding from the lower surface of the beam 50-100 nm, which when engaged produces a circular contact area. The two contacting areas are an evaporated thin-film layer of gold, forming the lower contact surface and a sputtered layer of gold, forming the underside of the beam.
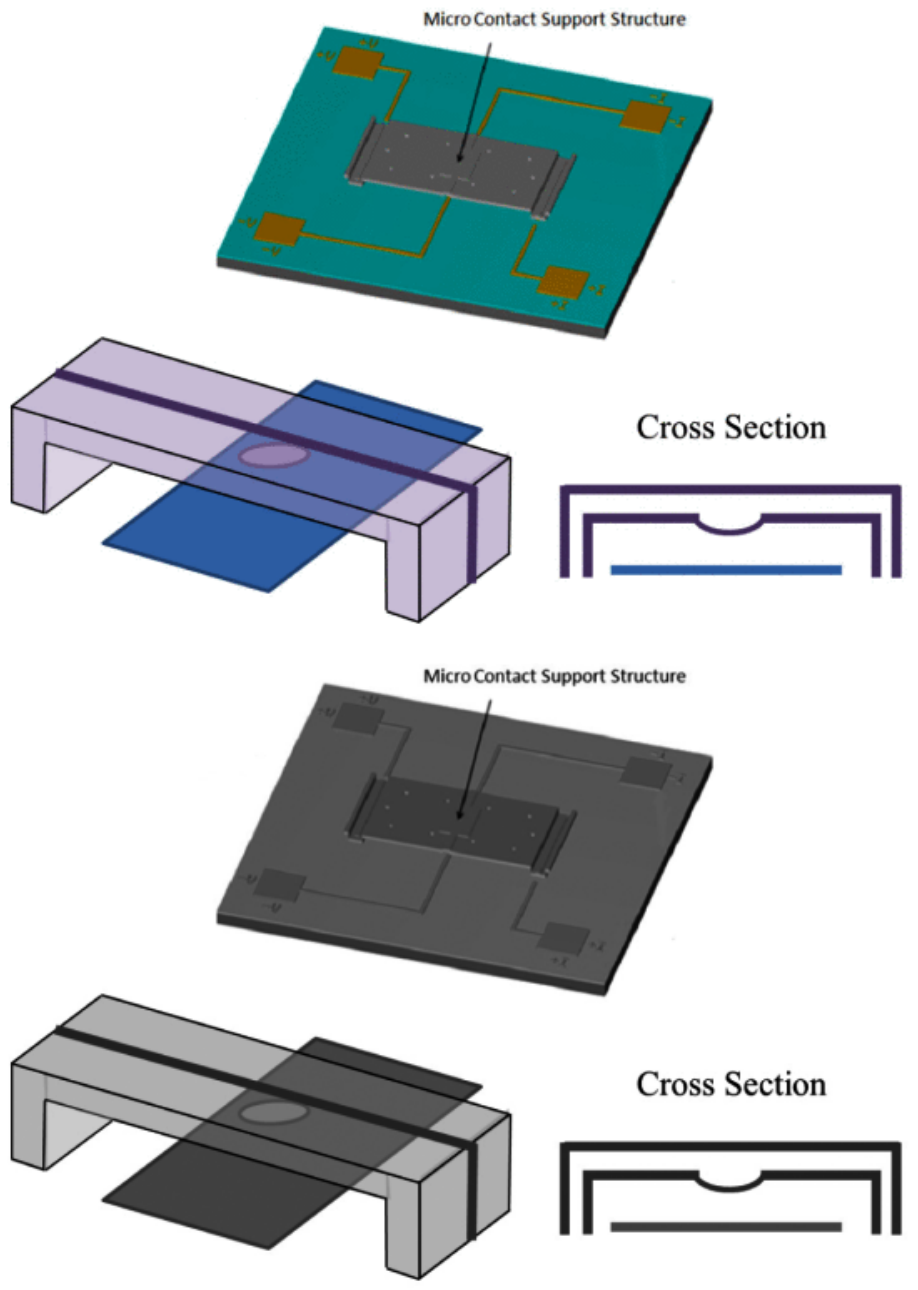

Fig. 2. Physical structure of microcontacts, fabricated with a hemispherical contact bump on lower side of the upper support beam.

To fabricate these devices, the silicon substrate was first isolated with either silicon oxide or silicon nitride. The bottom metal was patterned using $280 \mathrm{~nm}$ of evaporated gold on a 10-nm titanium adhesion layer. The beam itself was formed from patterning photoresist, which included the full shape 
of the beam, the two anchoring pillars, and an impression of the hemispherical contact bump. On this layer, gold was sputtered as the upper contact material. Finally, the beam structure itself was grown through electroplating. The final dimensions of the beam structure were $150 \mu \mathrm{m}$ in length, $75 \mu \mathrm{m}$ in width, $6 \mu \mathrm{m}$ thick, with a 1- $\mu \mathrm{m}$ separation between the upper and lower contacts. Contacts are released Microposit 1165 remover and transferred to methanol, which was then subjected to triplepoint $\mathrm{CO}_{2}$ drying and repeated plasma ashing both before and after wire bonding. These mounted and wired reticules were then subjected to a minimum of $12 \mathrm{~h}$ of nitrogen purging prior to any mechanical or electrical loading.

\section{Test Procedure}

Testing using this stand was accomplished by repeating two processes in succession: the measurement process and wear process.

The measurement process was conducted by applying a $1 \mathrm{~V} \mathrm{dc}$ load to the beam and slowing advancing the force sensor in 20-nm increments until contact was made between the sensor and the beam. The sensor continued to advance until current was detected through the contact and a voltage drop was observed. At this point, the force on the sensor reading was marked and treated as an offset, effectively resetting the force measurement to zero. The piezo actuator continued to advance until the desired contact force was obtained ( $200 \mu \mathrm{N}$ for all results presented here), all while recording the current through the microcontact, the voltage across it, and the force applied to it. A sample of one of these measurement cycles will be shown in (Fig. 12), but the final resistance, to which the contact settles, was used as the single reportable value when plotting contact resistance versus number of cycles. The contact resistance was calculated from these measured values using the Holm crossbar technique. ${ }^{6}$ This technique uses a known current flowing through the contact while measuring the voltage across the contact, and from these two values, the contact resistance was calculated. As the voltage measurement was performed with a high-impedance volt meter, this effectively isolated the voltage measured to the contact itself. This produces a detailed set of data relating contact resistance to contact force.

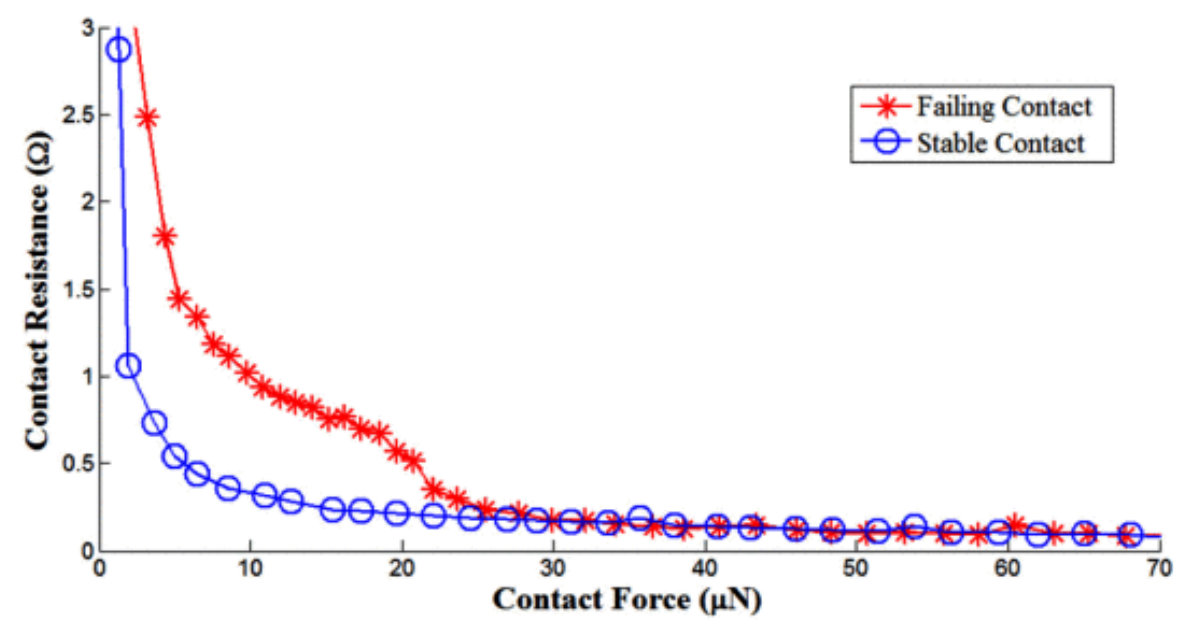




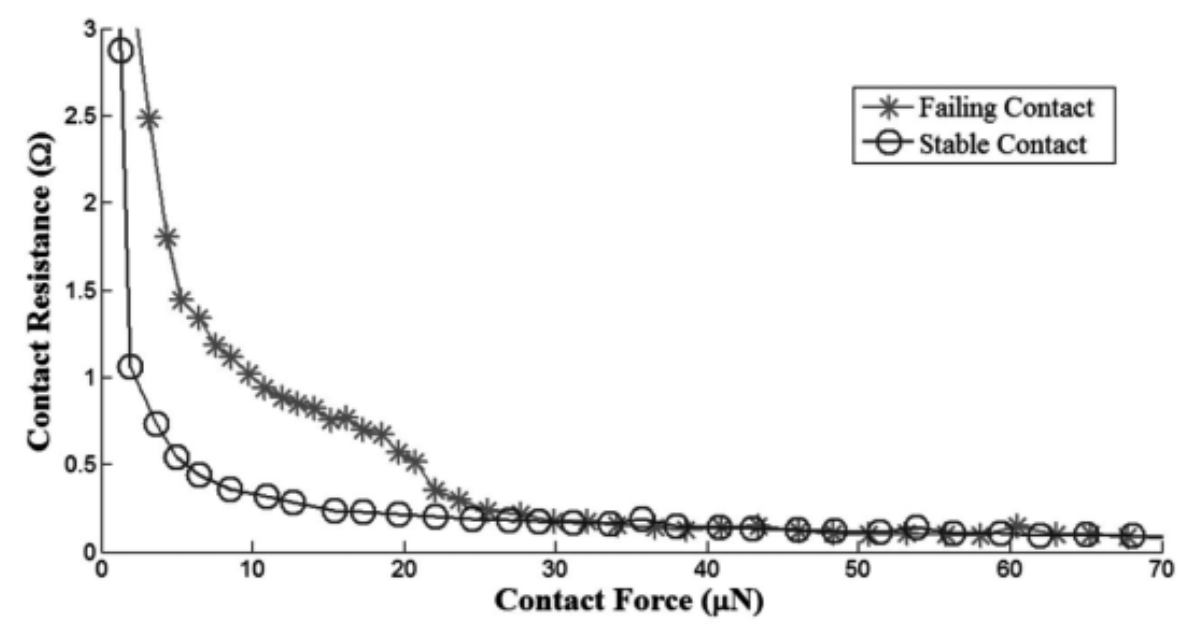

Fig. 12. Comparison of two single contact resistance measurements cycles, one at 10 million cycles for a device that remained stable, and the second shows a measurement shortly before the device failed, where it no longer conducted current upon closing.

The wear process was used to induce wear upon the contact in the shortest period of time feasible. Knowing the position required to achieve contact closure from the last measurement allowed for the piezo actuator to be positioned and cycled at high frequencies (up to $2.5 \mathrm{kHz}$ ) to impose repeated wear on the device. At the correct time during contact closure, a load was applied and then removed to simulate contact operation. The stand was designed for this load to be a low-voltage dc load, but this signal can be used as a waveform trigger to instead test with an ac load. Failure during this part of the process results in recording the number of cycles induced up to that point, along with the failure type (either failure to open or failure to close). Contact resistance data were not collected during this process.

The frequency of data collection can be run in two modes. For testing to a low number of cycles $(<\sim 100000)$, data can be collected repeatedly every certain number of cycles. However, this becomes impractical for longer tests as the test measurement process alone can take anywhere between 2 and 10 min per data point depending on the device and how it is set up. For a small number of measurements this is trivial, but becomes overwhelming at large numbers of cycles. Therefore, for devices tested to higher lifetimes, it becomes necessary to collect data a set number of times per decade. Note that measuring a set number of times per decade requires that as the devices ages, more cycles are imposed between measurements, which makes short-term changes difficult to detect. Balancing the desired number of cycles, the number of devices to be tested, and data resolution and accomplishing this in the time permitted require a tradeoff, which then must be accounted for when analyzing the results.

\section{Contact Cycling}

In previous experiments, devices of this design that were subjected to dc loads typically lasted well for past 10 million cycles. ${ }^{11}$ For all the tests conducted in this effort, each device was operated up to 10 million cycles or device failure, whichever came first. Data were collected such that three test points per decade were obtained (or a total of 21 test points over the life of a device) and was repeated for a variety of test conditions and with repetitive testing where feasible. Failure was recorded automatically 
and occurred in one of three forms. The contact remained closed from the last set of wear testing, the contact failed to conduct current after being closed, or the beam was not sensed as the force sensor advanced (typically indicating a failed sensor rather than failed device).

During the wear process, the test stand controlled both the oscillation of the force sensor and the timing of the load applied to the contact. Depending on the phase and duration of this load, the contact was subjected to either cold-switching or some version of hot-switching, as shown in Fig. $3 .^{9}$
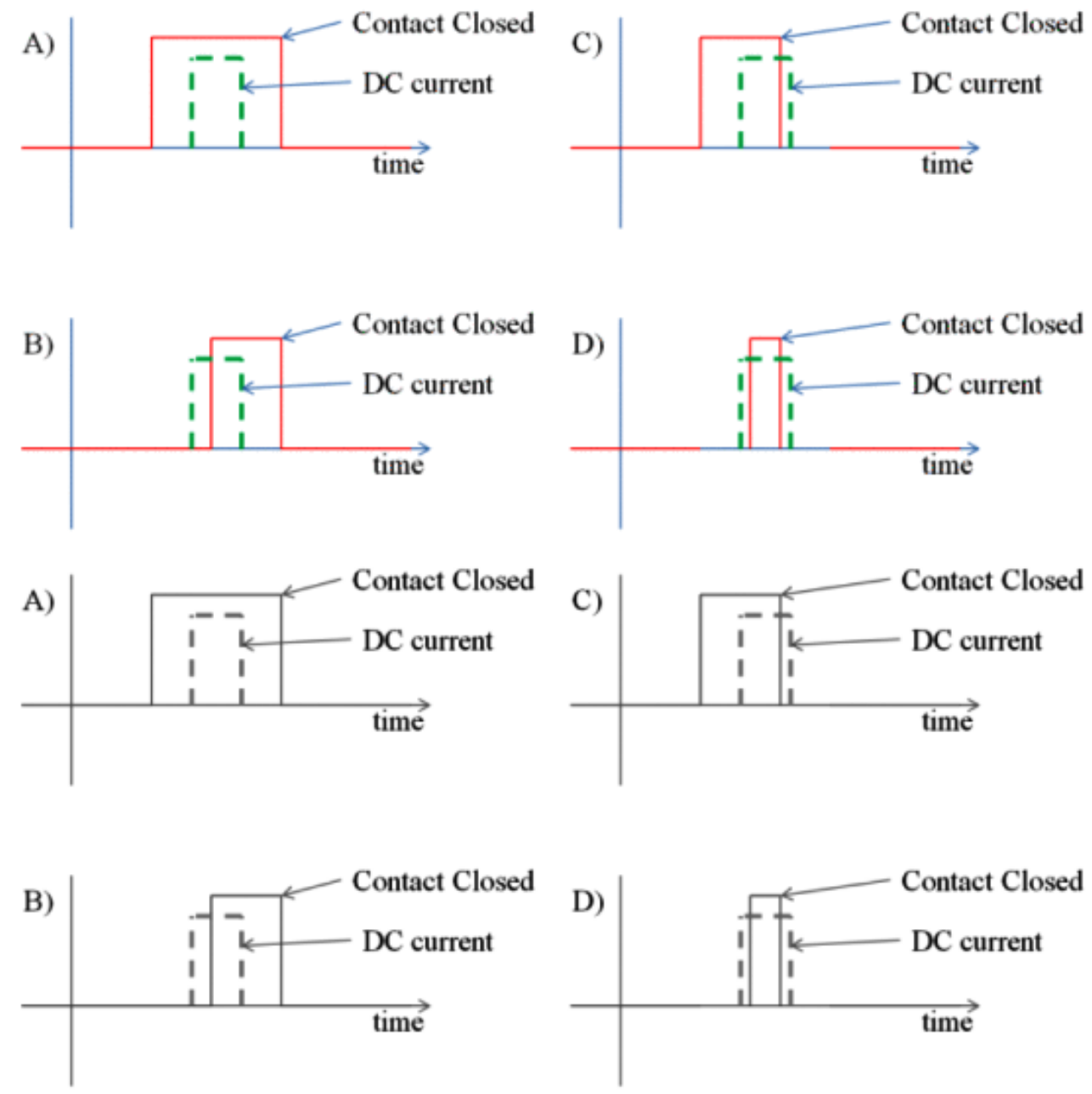

Fig. 3. Relative timing of contact closure (solid line) with respect to applied current (dashed line) can result in (A) cold-switching, (B) hot closure, (C) hot-opening, or (D) hot-switching.

Cold-switching implies that current flowed only through the contact while it was fully closed [see Fig. $3(\mathrm{~A})]_{.12}$ Hot-switching occurred when voltage was applied prior to closure, current was flowing through the contact while it was opened, or both were performed [see Fig. 3(B)-(D)]. When considering the testing results of low-frequency ac loads, this timing was critical. Recall the overall objective of testing considering not only the effects of hot-switching with an ac load, but also if ac loads affect performance on a cold-switching contact. To ensure that the correct test conditions are validated, we need to be mindful of the load frequency, how this load was applied (i.e., single burst, gated for a specific duration, etc.), and how this may limit the frequency at which we can cycle the contact. 


\section{Test Methodology}

\section{A. Low-Frequency AC Load Tests}

To determine what kind of loading was applied to the contact, we must consider the frequency of the load and how many cycles were applied, relative to the frequency at which the contact was cycled. The testing we focused on in this paper was explicitly low frequency. Therefore, the duration of even a single cycle of the load requires the switch be closed during its duration if we wish to maintain cold-switching conditions. In order to accommodate this timing requirement, the frequency at which testing could occur was limited. The relative timing of these events is shown in Fig. 4.
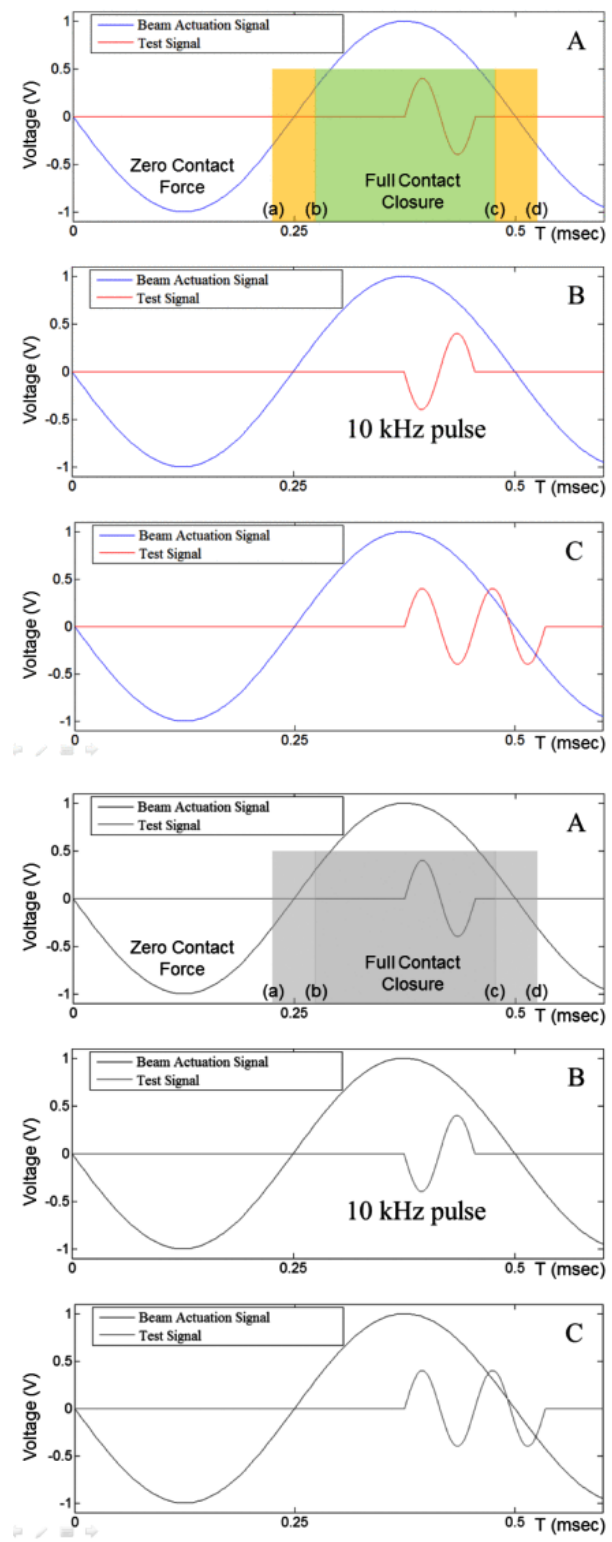

Fig. 4. Timing of test signals relative to beam actuation signal (voltage versus ms). (A) Single coldswitching cycle was induced, starting with unfavorable polarity for half the cycle and favorable for the second half, where load was applied within the window of contact closure [between points (b) and (c)]. Point (a) represented where the contact gap closed and contact was made, but at zero contact force, 
and between points (c) and (d), the contact was opening. Outside these regions, the contact was opened fully. (B) "Inverted" signal. (C) Extended signal that intentionally induces ac hot-switching conditions.

In Fig. 4(A), a test frequency of $2 \mathrm{kHz}$ was used, which required a beam actuation signal as shown in relation to a single cycle of a $10-\mathrm{kHz}$ pulse. As the beam actuation signal oscillated, contact was made at point (a) in Fig. 4(A). Contact force continued to increase until point (b) in Fig. 4(A), where we considered the contact fully closed. The exact width of this region of full closure depends on precisely how far the force sensor was from the device during test setup, but normally was controlled to approximately this range of closure. The force continues to increase and reaches its maximum (200 $\mu \mathrm{N})$ when the beam actuation signal peaks, and then decreases once again to point (c) in Fig. 4(A). Lightly loaded contact closure occurs between points (c) and (d) in Fig. 4(A), and outside this region, the contact is open. This means that during full contact closer, the contact force does vary, however, as will be shown in Section IV (Fig. 12), a stable contact of this design reaches a value close to the steady-state value at only $\sim 30-50 \mu \mathrm{N}$, with a very minimal change in contact resistance beyond this contact force.

The second condition [see Fig. 4(B)] shows the same two relative frequencies but with an inverted load. The third case [see Fig. 4(C)] demonstrates trailing edge hot-switching due to a second cycle imposed on the load and that the contact cycling frequency remains unchanged.

These distinctions were not only relevant because the contacts were hot- or cold-switched, but also because they experienced current flow in both directions while contact closure occurred. When testing a contact under dc conditions, it has been demonstrated that two identical contacts tested in the same manner but with an opposite polarity on the applied load can drastically affect the longevity of the contact, ${ }^{10}$ even if both contact materials are similar materials, such as gold/gold, as relevant properties such as hardness and elasticity vary depending on the method of deposition of the two contacting layers. If we then subject a contact to hot-switching conditions as in Fig. 4(C), the contact is thus subjected to repeated reverse biasing in addition to any potential damage caused by hot-switching the device. $^{8}$

This then leads us to consider how to mitigate damage caused by this manner of operation. To accomplish this, we investigated external circuit loading effects and specifically if certain configurations may aid in prolonging device life. ${ }^{13}$ We will start with considering that these external circuit loading effects perform under dc conditions.

\section{B. External Circuit Loading Tests}

To investigate this concept, we will build on the work in, ${ }^{13}$ which theorizes on the effects of external inductance, capacitance, and resistance on microcontact behavior. To briefly summarize the theory, potential damage caused during leading edge hot-switching was due to transient charge on the contacting surface. During contact closure, this charge leads to regions of extremely high current density followed by contact closure over the weakened material. This charge results from the capacitive nature of a pair of contacts prior to closure, and any external capacitance in parallel with such a contact can in theory amplify the effect. ${ }^{13}$

Similarly, during trailing edge hot-switching, this theory predicts that damage results from the transient current flowing through a contact, which attempts to continue to flow after the contact begins to open, 
which can similarly lead to regions of extremely high current density. In this case, any external series inductance can similarly exaggerate these kinds of effects.

This work also postulates that these effects may be mitigated by applying these active components in the opposite manner (i.e., parallel inductance to mitigate preclosure surface charge and series capacitance to mitigate maintained current flow), or even if the addition of passive components could produce a similar effect by applying voltage and current limiting elements during operation.

To experimentally validate this theory, testing was conducted with the same test fixture and microcontact structures, but these structures were combined with other circuit elements as shown in Fig. $5 .{ }^{9}$ In Fig. 5, the boxed switch represents the microcontact being tested. For all inductors used in all testing, $100 \mu \mathrm{H}$ was used. All parallel resistances were $5 \mathrm{M} \Omega$, and all series resistances were $1 \Omega$. Capacitance values unless otherwise noted were $0.6 \mathrm{pF}$, except for a single series of tests, in which 1.8 pF was used.

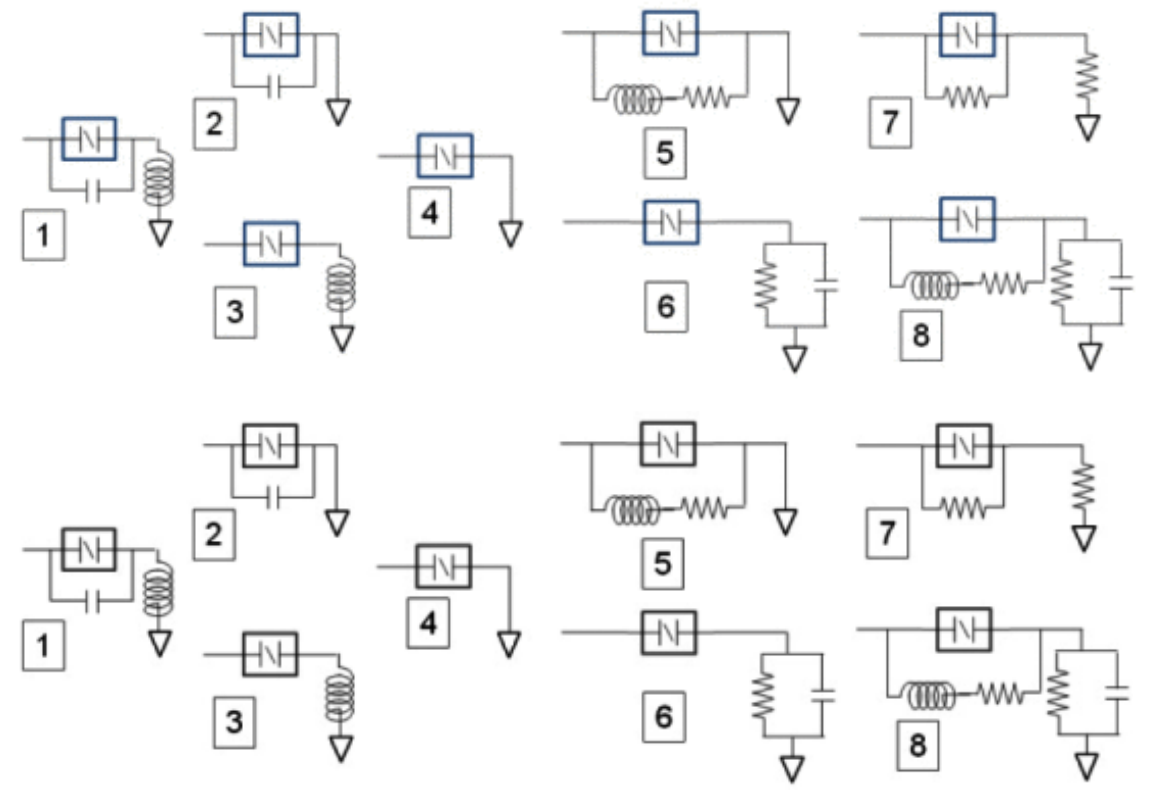

Fig. 5. Circuit configurations tested, all under dc load signal. 1-parallel capacitance with series inductance. 2-parallel capacitance. 3-series inductance. 4-baseline. 5-parallel RL load. 6-series RC load. 7-Resistive voltage division. 8-RLC protective loading. The inductance for these tests was $100 \mu$ $\mathrm{H}$, and the capacitance was typically $0.6 \mathrm{pF}$ except for one test at $1.8 \mathrm{pF}$. The resistance in parallel with the microcontact was $5 \mathrm{M} \Omega$, and the resistance in series with the microcontact was $1 \Omega$.

To isolate the effects caused by this kind of external loading, the eight circuit configurations shown were tested. In Fig. 5, configuration 4 will be considered the baseline-the contact by itself, with no parallel or series elements. While some measurements were conducted as part of these experiments, this configuration has been well characterized ${ }^{10,11,14}$ and the available devices were devoted primarily to other untested configurations. From the previously mentioned theory, the three circuits $(1,2$, and 3$)$ in Fig. 5 (left) are expected to result in worse overall performance for the reasons previously mentioned. The four circuits in Fig. 5 (right) (5-8) are expected to provide some level of damage mitigation. Circuit 7 is the purely resistive circuit configuration. 


\section{Low-Frequency AC Tests With Circuit Loading Mitigations}

Finally, we will bring these two concepts together and investigate if damage can be mitigated when using these kinds of microcontacts under low-frequency ac loads through external loading. To test this, four circuit configurations were tested, each with three different frequencies of the applied loading, and the results compared. Reactive elements were small enough to keep any charge/discharge times for any of these components fast compared to either the applied loads or cycling rates of the devices. For resistive loading, series resistance was kept small and parallel resistances large for two reasons. First, it better simulated microcontact utilization in real-world applications, and second, it was required for accurate testing. To be able to detect a change in contact resistance during closure, a parallel resistor must be sufficiently large, and to ensure that adequate current was going through the contact to produce a valid measurement, any series resistor must be small enough to produce a sufficient change in voltage across the contact.

\section{Results}

\section{A. Low-Frequency AC Load Tests}

The first series of tests was to evaluate the performance of ac loads without external circuit considerations. These were conducted under all three conditions outlined in Fig. 4, the first of which was a single cycle pulsed during each contact closure. Four frequencies were tested, and for each of these frequencies, the cycle time of the contact was slowed to ensure that only cold-switching conditions existed as mentioned above. Therefore, for the 100- and 10-kHz loads, contact switching occurred at 2 $\mathrm{kHz}$; for a 1-kHz loading, this cycle frequency was slowed to $200 \mathrm{~Hz}$; and for a $100-\mathrm{Hz}$ load frequency, the cycling frequency is limited to $20 \mathrm{~Hz}$. Fig. 6 shows the results of the life of the devices tested at these four frequencies.

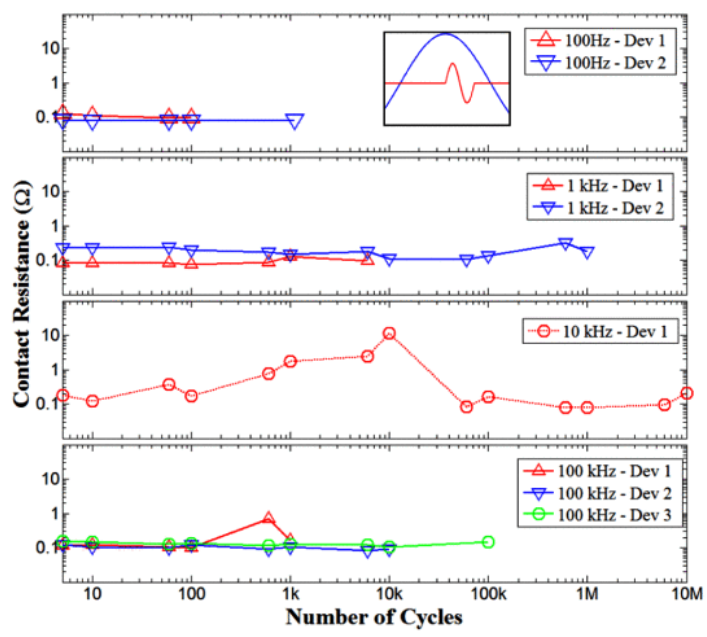




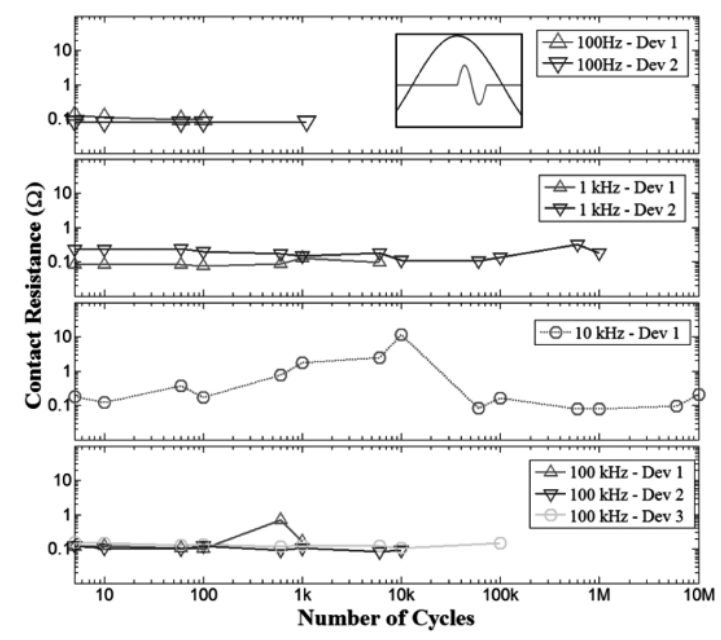

Fig. 6. Contact resistance versus induced cycles with an ac load with signal frequencies of $100 \mathrm{~Hz}, 1 \mathrm{kHz}$, $10 \mathrm{kHz}$, and $100 \mathrm{kHz}$ applied to cold-switching Au-Au microcontacts with an $8-\mu \mathrm{m}$ contact radius.

For all except one device, failure occurred prior to reaching the goal of 10 million cycles. For both the 1and $100-\mathrm{kHz}$ loads, the devices reached only 1000 cycles, while the 100-Hz devices both failed much sooner. For these seven cases, failure was due to the contacts becoming permanently closed without an applied load (i.e., shorted and closed).

The next tests explored the effects of intentionally extending the load such that hot-switching was induced with an ac load. In addition to a 1-kHz load frequency, two additional tests were run with slightly lowered frequencies $(800$ and $600 \mathrm{~Hz}$ ) to slightly extend the timing of the effect relative to the contacts opening. The results of these three conditions are shown in Fig. $7(\mathrm{~A}) .{ }^{15}$

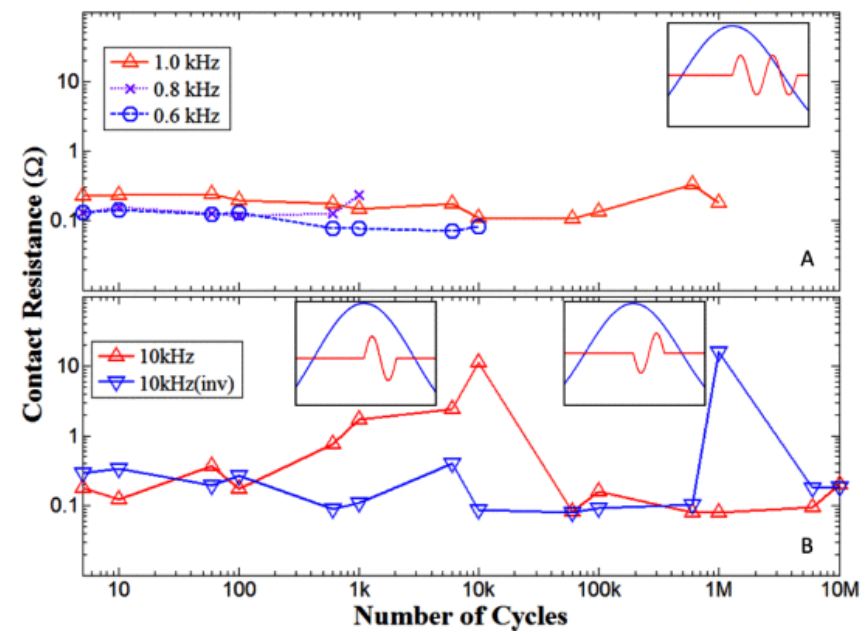




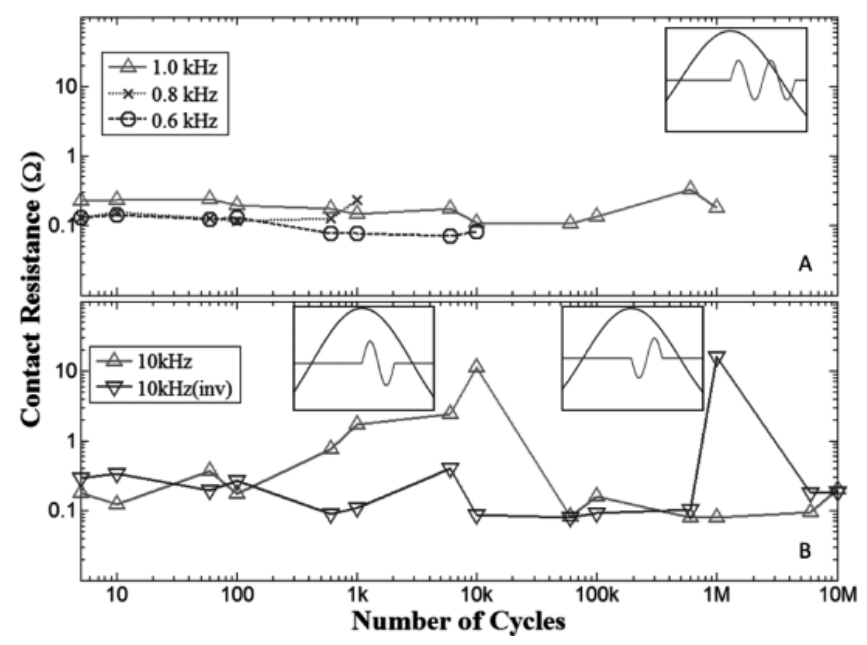

Fig. 7. (A) Comparison of hot-switched ac loads of frequencies $1 \mathrm{kHz}, 800 \mathrm{~Hz}$, and $600 \mathrm{~Hz}$ where all three devices eventually failed to open. (B) Comparison of cold-switched loading of inverted and noninverted single cycles, $10-\mathrm{kHz}$ loads applied to an $8-\mu \mathrm{m}$ contact radius of Au-Au microcontact cycled at $2 \mathrm{kHz}$.

The next test conducted was similar, except for the load being inverted [recall Fig. 4(B)]. For this test, the frequency selected was $10 \mathrm{kHz}$, which provided the best results in the first set of testing. Fig. $7(B)^{15}$ shows both the noninverted results from the first test and the results of the inverted test. This device also lasted for 10 million cycles and showed little difference compared to the noninverted tests.

If we bend back the upper beams for some of these failed devices, we can investigate the damaged surfaces with a scanning electron microscope (SEM), as shown in Fig. $8 .{ }^{15}$

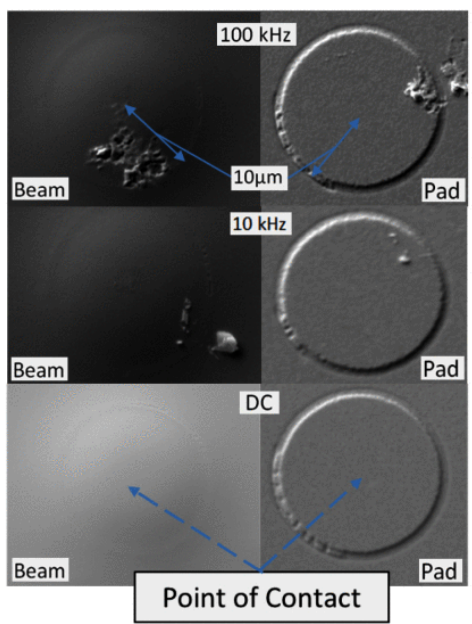




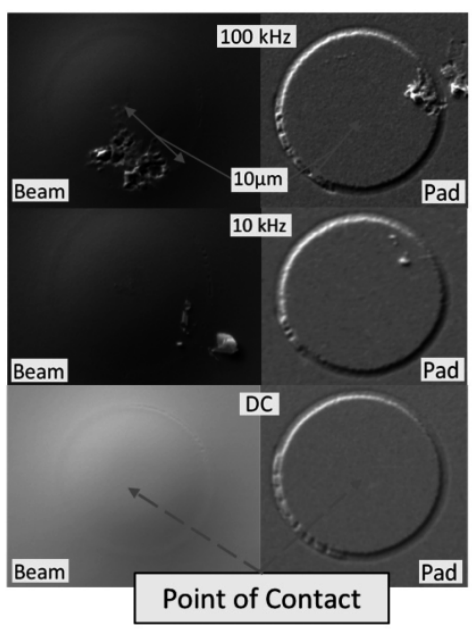

Fig. 8. Exposed contacts after folding back the upper beams (left) and their corresponding lower pads (right) from three devices exposed to cold-switched loads, at $100-\mathrm{kHz}$ and $10-\mathrm{kHz}$ ac loads (as labeled), compared to an identical device tested with cold-switched dc to $10 \mathrm{M}$ cycles of operation. The circular shape on the pad side is an intentional conformal feature in the bottom evaporated gold layer, which marks the corresponding outer radius of the mating bump (in which the same circular shape can be seen, but is diminished during the photoresist reflow process, which gives the upper beam contacts a hemispherical shape.

Fig. 8 (left) shows the hemispherical bump discussed previously, and the profile of this feature becomes apparent in the shadowing of the surface. Fig. 8 (right) shows the corresponding lower contact surfaces. The two images in Fig. 8 (top) are from the $100-\mathrm{kHz}$ device from the first test series and the two images in Fig. 8 (middle) from the 10-kHz device from same tests. The two images in Fig. 8 (bottom) are from the previous device subjected to dc testing. ${ }^{10}$ This unloaded dc device showed stable performance up to 10 million cycles, at which point testing was halted so that the device could be examined to look for any signs of wear. Note that the failed devices (in some cases with only a few thousand cycles) showed significant damage due to material being transferred between contacts, with pits where material was removed and the debris produced in some cases still evident. In both cases, the contact location on the lower surface (which was the center of the patterned circle) shows very little signs of wear, indicating that the damage was inflicted primarily to the sputtered upper contact and not the evaporated lower contact.

\section{B. External Circuit Loading Tests}

To discuss the results of external loading on microcontact performance, recall the eight circuits presented in Fig. 5. These circuits were ordered by expected performance, where circuit configurations 1-3 incorporated all the elements, which were expected to be detrimental. Circuit 4 was the baseline with no external elements. Configurations 5, 6, and 8 included reactive elements, which were theorized as beneficial configurations, and circuit configuration 7 was purely resistive. A single baseline was run (circuit four) to ensure that the performance was comparable to the results from previous studies conducted with this design. In $\sim 40$ devices previously tested under identical baseline conditions, typical contact resistance between $\sim 0.1$ and $2 \Omega$ was common, with greater variance under 1000 cycles 
occasionally occurring as the device was worn in. ${ }^{10,11,14}$ For the other seven circuits, two devices were tested with each setup.

First, consider the three detrimental circuit configurations (1-3). The worst of these three circuits was the circuit that contained an inductor in series with the microcontact. While the other two configurations failed well short of the 10-million-cycle goal, series inductance failed much sooner. Fig. $9^{9}$ shows a comparison of the two devices tested in this configuration with a 1-VDC load, compared to the baseline device (circuit configuration 4), which lasted for 10 million cycles and was still functional at the point of test termination.
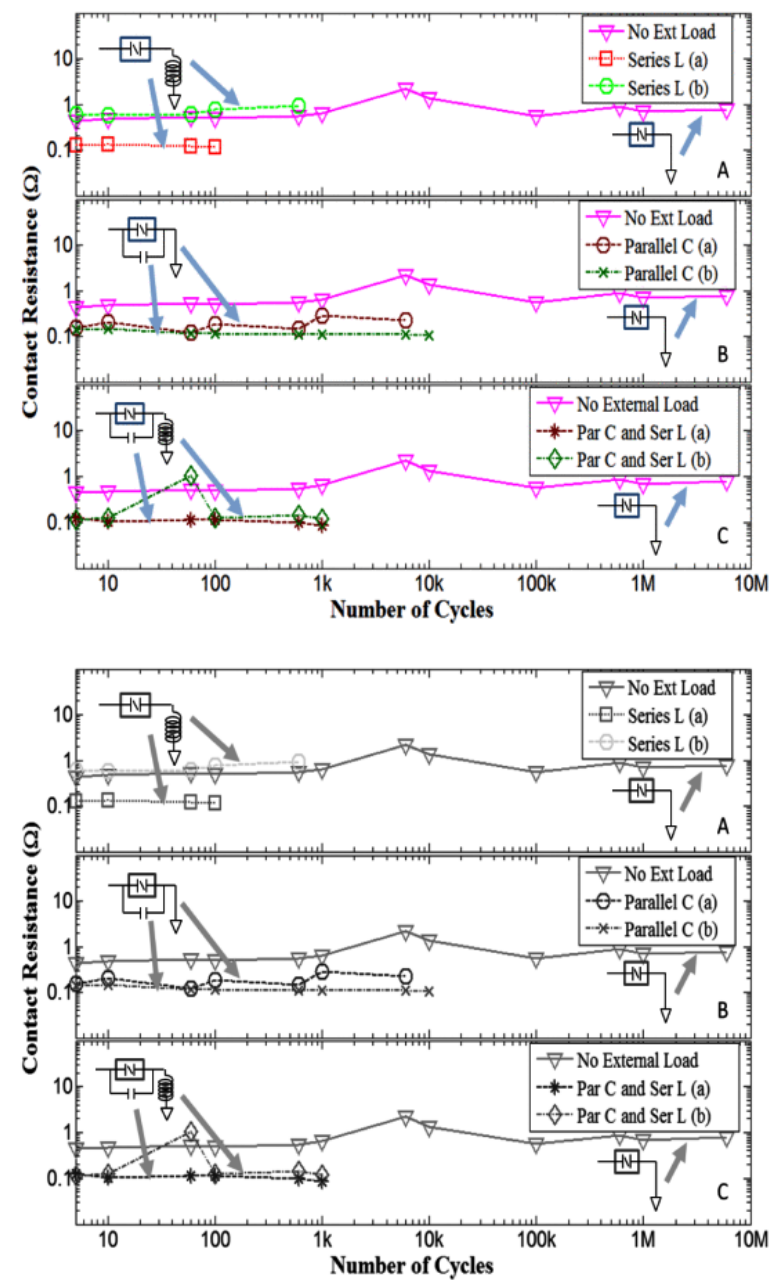

Fig. 9. Comparison of the contact resistance of $\mathrm{Au}-\mathrm{Au} 8-\mu \mathrm{m}$-radius microcontacts with three detrimental circuits. (A) Series inductance of $100 \mu \mathrm{H}$ was added. (B) Parallel capacitance of $0.6 \mathrm{pF}$ was added. (C) Both elements were added. All the six devices were tested with a 1-V cold-switched dc load, and all failed due to shorting at the number of cycles shown.

Next, consider the three reactive damage mitigating circuits from Fig. 5 (configurations 5, 6, and 8). For all three of these tests, all devices tested lasted to the 10-million-cycle goal and were still functional upon test termination. The configuration in which both devices appear to be the most stable was the 
series $R C$ configuration (see Fig. 5, circuit 6). The comparison of the two devices tested in this configuration along with the same unloaded element as shown previously is shown in Fig. $10{ }^{9}$
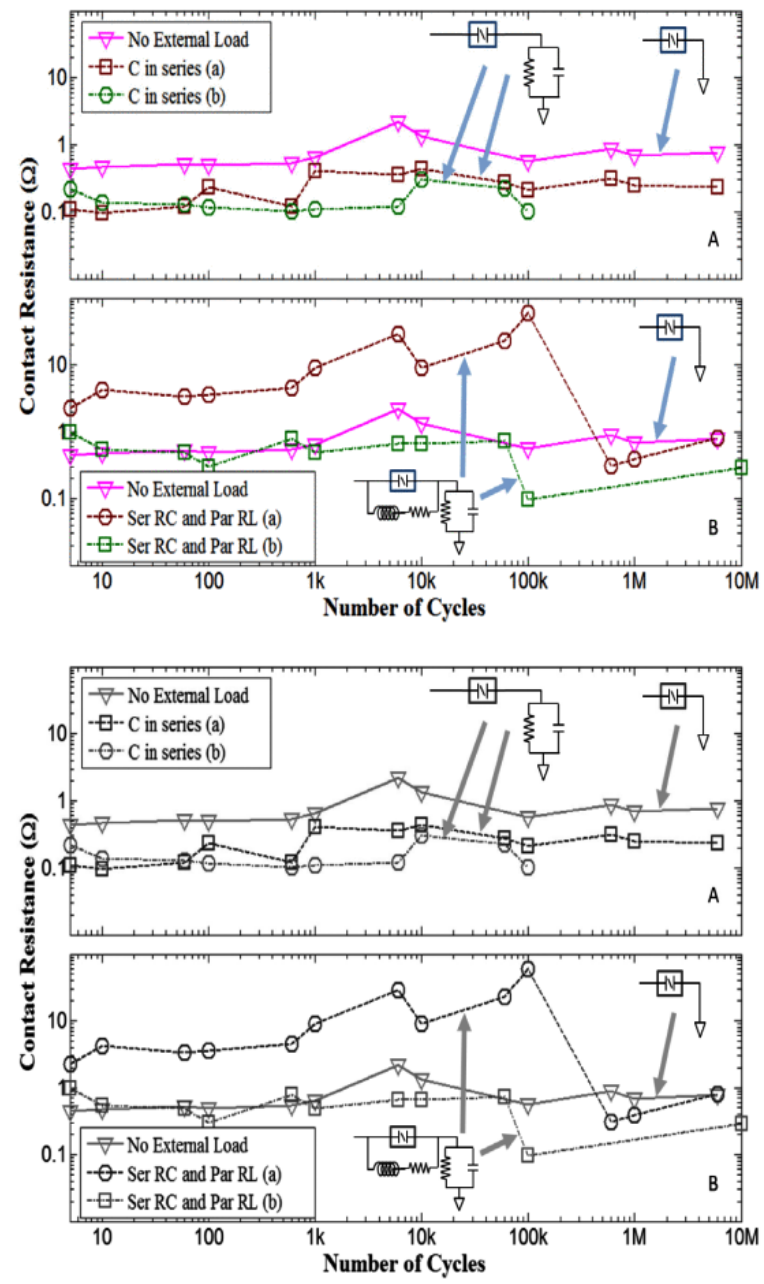

Fig. 10. Comparison of the contact resistance of unloaded $\mathrm{Au}-\mathrm{Au} 8-\mu \mathrm{m}$-radius microcontacts with two protective circuits. (A) Two series $R C$ external loads (a) and (b). (B) Both series $R C$ and parallel $R L$. The one failed device was due to shorting, and the rest all lasted to the target $10 \mathrm{M}$ cycles. The external resistor used was $1 \Omega$, the capacitor was $0.6 \mathrm{pF}$, and inductor was $100 \mu \mathrm{H}$.

While some variance was still present in the contact resistance, the reactive element circuit devices all recovered from any of these fluctuations and were able to "dial in" to a final contact resistance very effectively. Next, consider the purely resistive configuration as shown in Fig. $11 .^{9}$ 

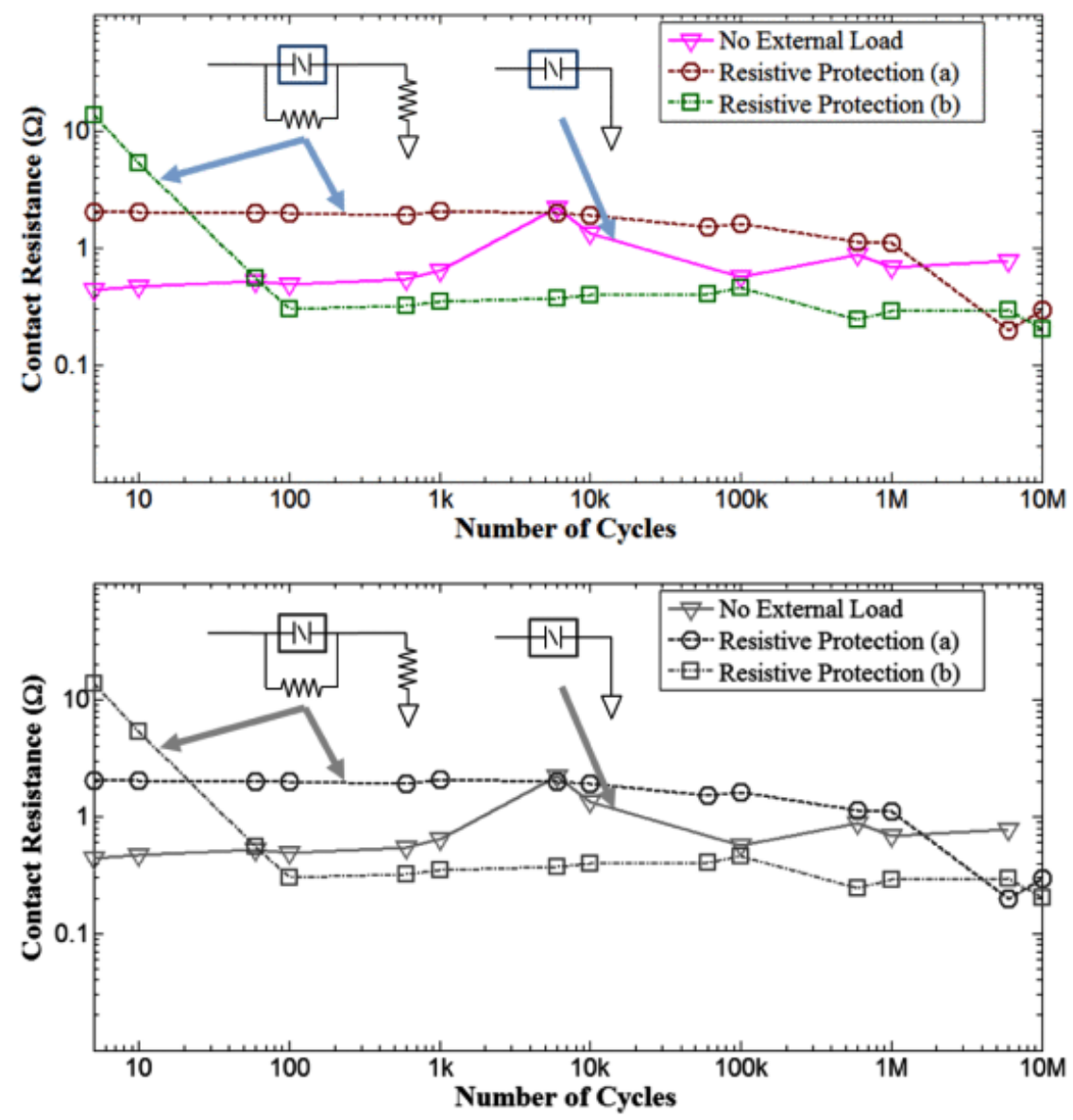

Fig. 11. Comparison of the contact resistance of $\mathrm{Au}-\mathrm{Au} 8-\mu \mathrm{m}$-radius microcontacts showing an unloaded device with both a $5-\mathrm{M} \Omega$ parallel resistance, capable of immediately dissipating any charge during contact opening, and a series resistance of $1 \Omega$, providing the ability to limit current while the contact was closed.

Recall Section II-C, where the measurement process was described for these devices. As the force sensor assembly was advanced and applied an ever-increasing external force to the upper beam of the contact support structure, contact resistance was measured at each step providing a detailed profile of contact resistance versus contact force. If we look closely at data collected from a device that failed prematurely, we observed that the last few measurements taken showed signs of the impending failure. To illustrate this, consider Fig. $12 .^{9}$

The two curves in this plot show data from two different devices, one that lasted for $10 \mathrm{M}$ cycles without incident ("stable contact" in Fig. 12) and one that failed before that point ("failing contact" in Fig. 12). This particular measurement is from device (b), which is parallel capacitance, in Fig. 9(B). This is the data from the measurement taken at $\sim 1000$ cycles (two measurements before failing at $\sim 10000$ cycles), which is when this abnormal curve first appeared. The second device shown in Fig. 12, labeled "stable contact," was the last test measurement from a device that had reached 10 million cycles and was still operational. The variation between the smooth exponential decay of the stable device and the irregularity in the failing device may prove to be a viable method to monitor the "health" of these devices during operation. 
As in the last set of experiments, one of the contacts was imaged using SEM imagery. The device selected had purely resistive protection and was investigated after it appeared to recover and settle [device (a) shown in Fig. 11]. The SEM results are shown in Fig. $13 .{ }^{9}$ In Fig. 13 (bottom and top), an expanded view of the larger image is provided of a beam folded back with a probe, and close-up views of both sides of the microcontact are provided, respectively. This was the same method that was used to image the contacts shown in Fig. 8. While there were signs of transferred material, note that this material does not have the same jagged appearance as the failed devices from Fig. 8 . While there was one small spot of material on the bottom of the contact bump, overall this image shows little sign of damage.
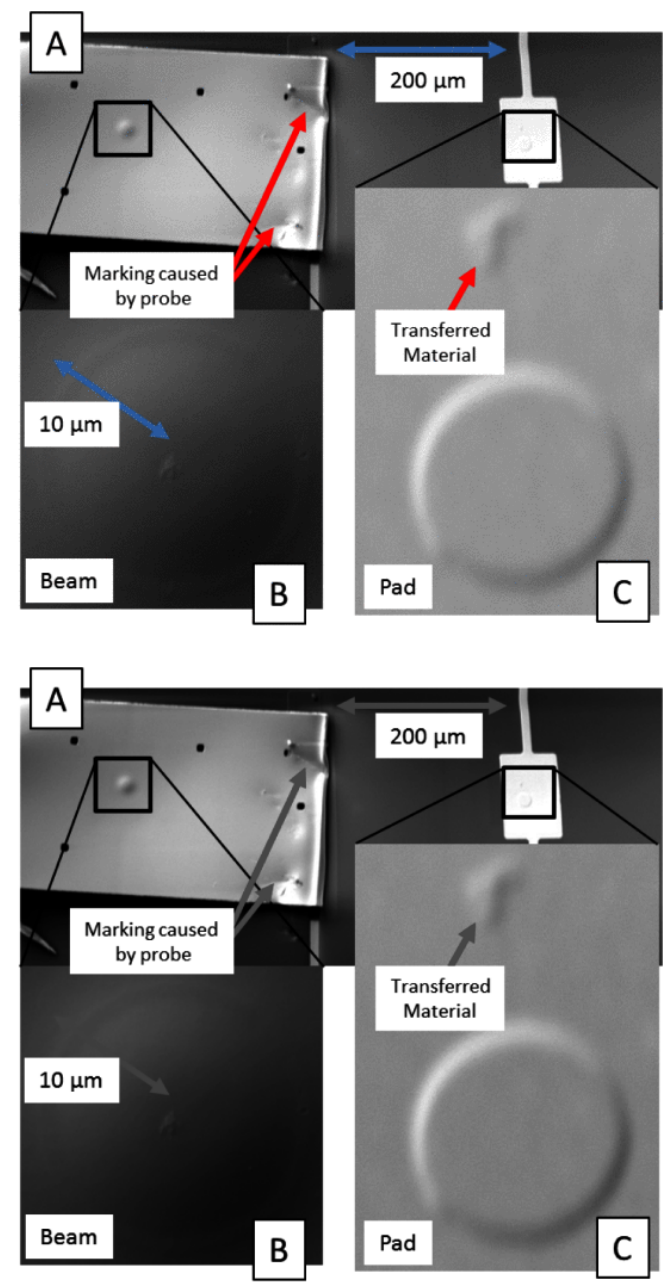

Fig. 13. SEM investigation of contact surfaces after testing to $10 \mathrm{M}$ cycles with a dc cold-switched load to investigate level of induced wear by folding the upper contact beam back after separating from the anchor. This device was tested with passive external circuit loading, with a $50-\mathrm{M} \Omega$ parallel resistance and a $1-\Omega$ series resistance.

\section{Low-Frequency AC Tests With Circuit Loading Mitigations}

Finally, we investigated the effects of combining this idea of protecting a circuit through external loading, and tested devices with these external circuits while exposing them to the same ac loads as we used earlier and observed if we can get improved results. 
For this series of tests, we used three frequency loads: $-1,10$, and $100 \mathrm{kHz}$. For each, the cycle rate during testing was adjusted to ensure that only cold cycling conditions were applied. For each of these three frequencies, devices were tested with each of the four protective circuit configurations shown in Fig. 5, parts 5-8, and additionally for the series capacitance circuit configuration, two different capacitances were investigated for a total of 15 tests conducted.

Of the 15 tests ran, 13 devices ran to 10 million cycles and were still operational. The first failed test was the 1-kHz load using parallel inductance, which failed at just over 8000 cycles. The second premature failure was also with a 1-kHz load but with both series capacitance and parallel inductance and failed at 4.88 million cycles. All three of these failures were due to contact shorting during the wear cycling. All other tests showed very little variation in contact resistance early in testing, until just after 100000 cycles. At that point, in almost every case, the contact resistance began to climb with device life. The results from the purely resistive test are shown in Fig. 14.
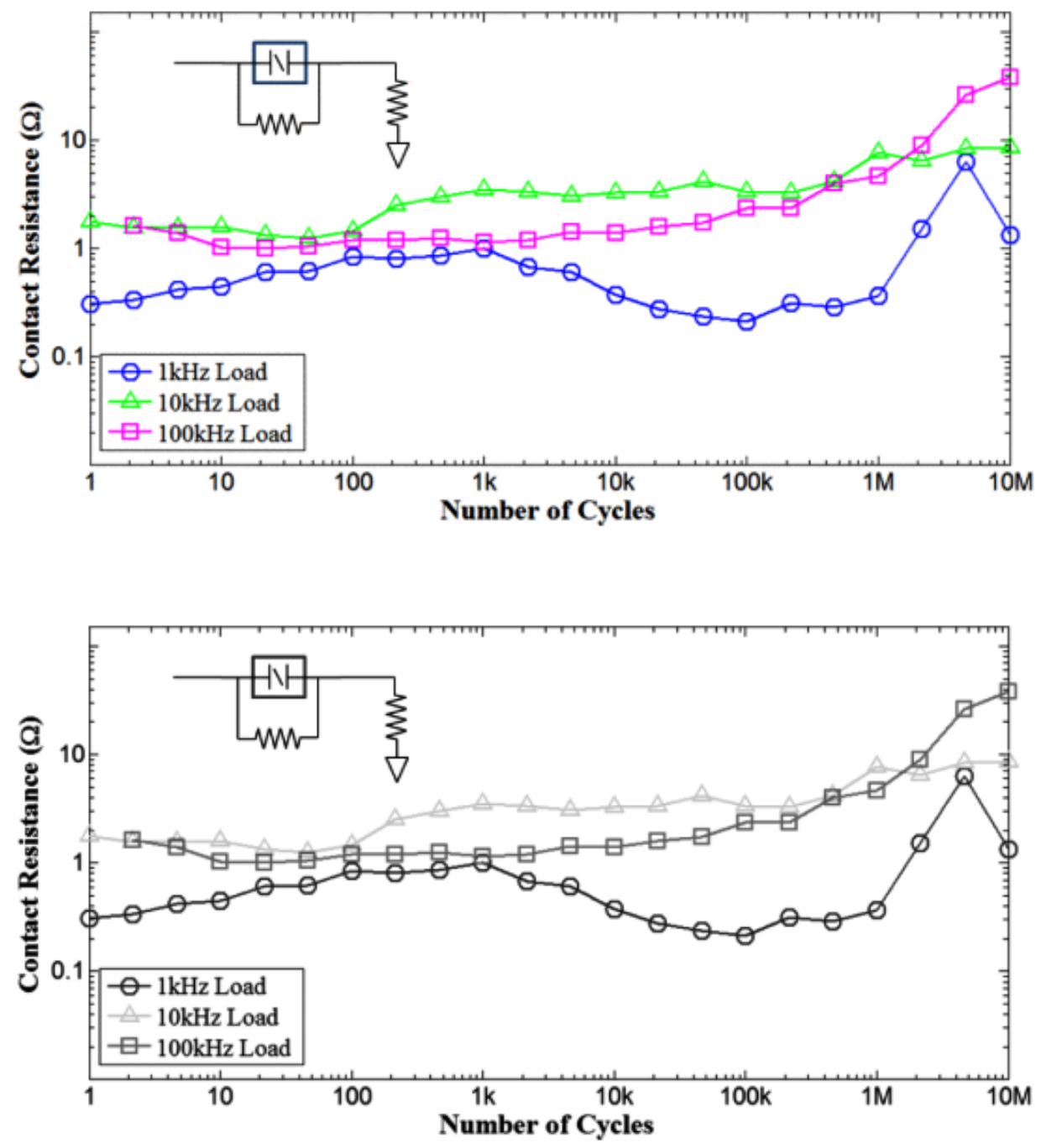

Fig. 14. Comparison of contact resistance versus device life of Au-Au 8- $\mu \mathrm{m}$-radius microcontacts under the three ac loads shown, all cold-switched with a parallel 5-M $\Omega$ resistor and a 1- $\Omega$ series resistance added externally. All three devices remained operational at the 10-million-cycle target. 
The next two tests involved the addition of parallel resistive loading along with series capacitance. Both tests used a $5-\mathrm{M} \Omega$ parallel resistance and a $1-\Omega$ series resistance, but with two different capacitances (0.6 and $1.8 \mathrm{pF}$ ). The results are shown in Fig. 15.
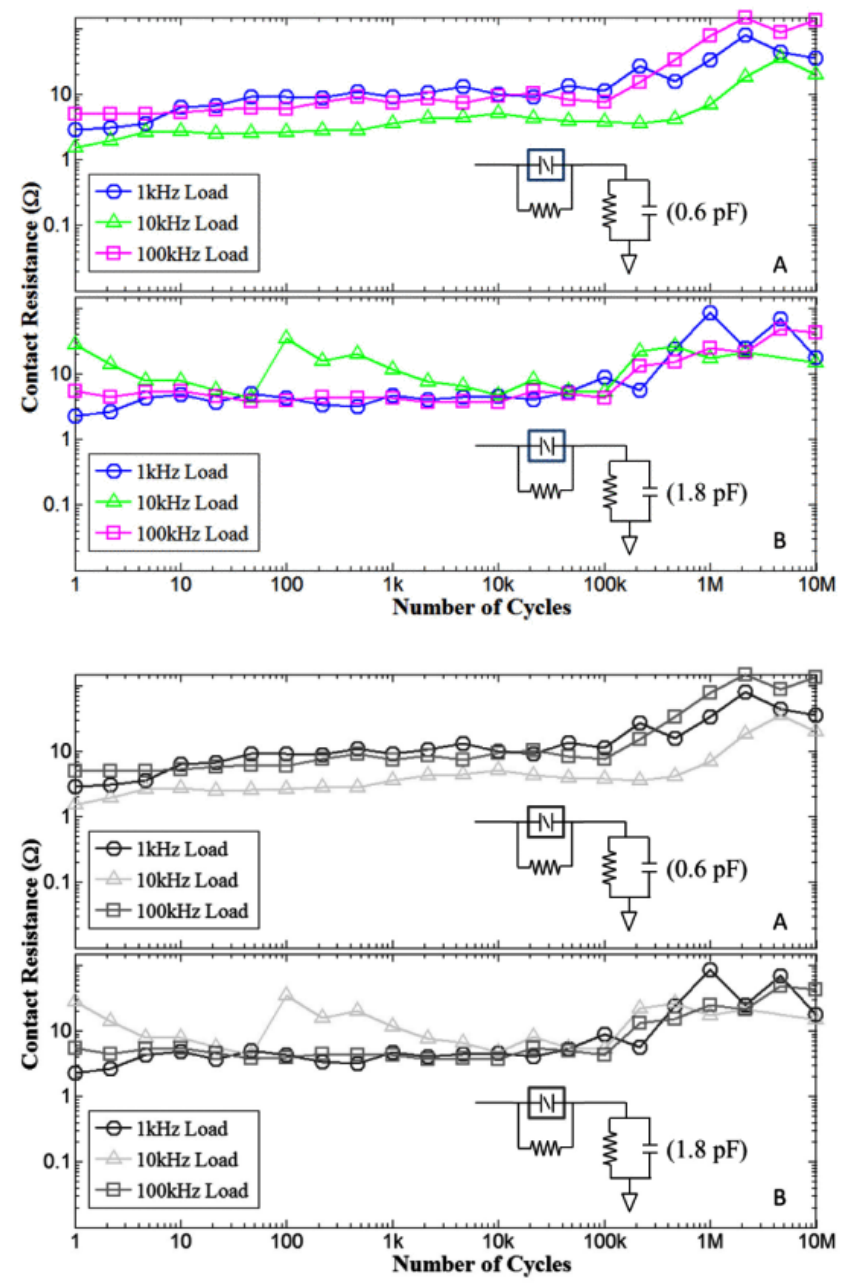

Fig. 15. Comparison of contact resistance versus device life of $\mathrm{Au}-\mathrm{Au}$ 8- $\mu \mathrm{m}$-radius microcontacts for two test circuits (both with a 5-M $\Omega$ parallel resistance and an $R C$ circuit in series, using a $1-\Omega$ series resistance, along with two different-sized capacitances as shown). The tests were conducted at the three frequencies listed, all of which were still operational at the 10-million-cycle target.

Finally, reactive protection was tested, which not only utilized series capacitance but also parallel inductance. The same resistances were used as in the last two tests, the series capacitor was $0.6 \mathrm{pF}$, and the parallel inductance was $100 \mu \mathrm{H}$. The results of these tests are shown in Fig. 16. 

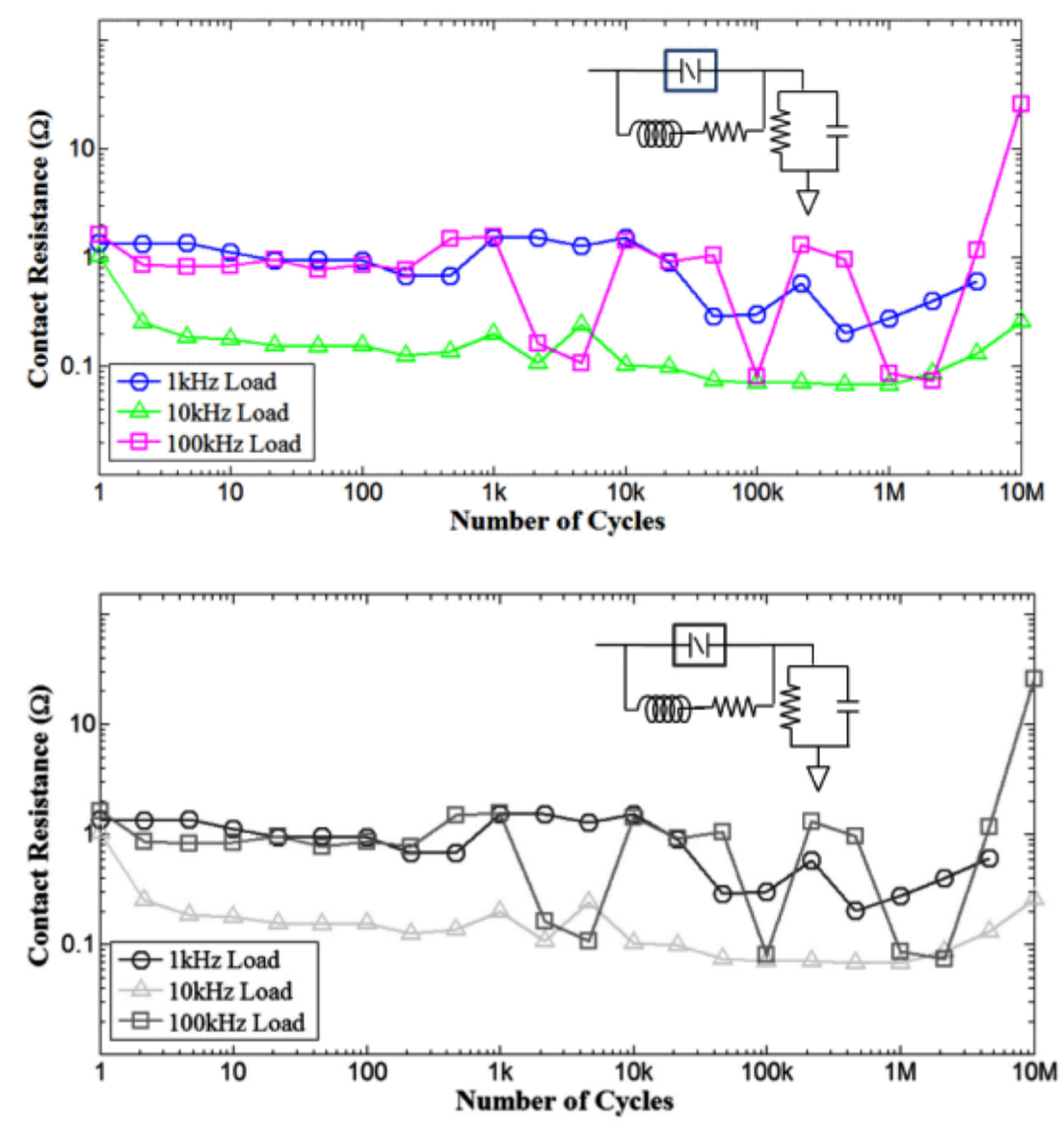

Fig. 16. Comparison of contact resistance versus device life of Au-Au 8- $\mu \mathrm{m}$-radius microcontacts for the test circuit shown. The inductance used was $100 \mu \mathrm{H}$ along with a 5-M $\Omega$ parallel resistance and an $R C$ circuit in series, using a $1-\Omega$ series resistance, and $0.6 \mathrm{pF}$ of capacitance. The tests were conducted at the three frequencies listed, two of which were still operational at the 10-million-cycle target and the other that failed to close just prior to reaching 10 million cycles.

\section{Conclusion}

From these results, several conclusions can be drawn by stepping through the experiments performed, considering the incremental changes applied between tests and evaluating the results of these changes.

First consider the history of performance of these devices under dc loads with no external loading. While a few representative devices were tested with each test series presented here, if we look at data collected over the past several years, in $\sim 40$ devices previously tested under identical baseline conditions, the typical contact resistance between $\sim 0.1$ and $2 \Omega$ s was common, with greater variance under 1000 cycles occurring as the devices were worn in. Of these 40 devices, the failure rate prior to 20 million cycles was $\sim 5 \%$. These data were taken with devices fabricated at the same time (and in some cases, on the same wafer) as the data collected here, so based on this performance record, it was assumed that if there was significant premature failure, it was due to test conditions imposed and not inherent in the devices themselves. 
Next, consider the results of replacing the dc load with ac. Due to the limited number of tests for frequency inversion and hot-switching, it would be unreliable to draw too many correlations to specific frequencies, but overall for all three devices tested under hot-switching conditions, all three failed prior to the 10-million-cycle target. Under cold-switching conditions, however, of the eight devices tested, only one survived to the 10 -million-cycle point, which overall was an $87.5 \%$ failure rate. SEM imagery confirmed that comparing devices that failed under these ac loads showed substantial damage compared to identical contacts exposed to 10 million cycles of dc loading and were still operable.

Following this was the investigation of the effects of external circuits on microcontact performance under dc loading conditions. The theory predicted that series inductance and parallel capacitance can accelerate premature failure in microcontacts, which was validated in all six of the test devices, which failed prematurely in these destructive circuit configurations. Conversely, it was expected that series capacitance and parallel inductance should aid in device performance. Three of the four devices tested under these conditions lasted for the full test duration. Additionally, passive protection also behaved as expected, and both devices tested again lasted for the full 10 million cycles.

This culminated into the investigation of mitigating the failure rate of ac load tests by applying external protective circuit elements. Under test conditions, which were identical to those used in the initial ac testing, of the 12 devices tested, only one failed. In other words, applying external circuit protection reduced the failure rate from $87.5 \%$ to $8.3 \%$. As before, drawing specific conclusions on which configurations performed better than others may not be substantiated, given the limited number of tests for each circuit, but when the entire data set was considered, these more general observations are apparent.

One side observation is that when investigating the detailed data collected during a single contact resistance measurement, the "smoothness" of the resistance-force curve produced a distinctive behavior in devices that failed shortly after producing these abnormal measurements. By evaluating a deviation from the ideal, smooth curve that the contact resistance theory predicts, one indicator of premature contact failure becomes available.

\section{Acknowledgement}

The views expressed in this paper are those of the authors and do not reflect the official policy or position of the U.S. Air Force, Department of Defense, or the U.S. government.

\section{References}

1. V. S. Cortes, G. Fischer, "Shunt MEMS switch requirements for tunable matching network at $1.9 \mathrm{GHz}$ in composite substrates", Proc. GeMiC, pp. 422-425, Mar. 2015.

2. A. K. Chaurasia, R. Mehra, "Robust design of RF MEMS switch design with reduced buckeling effect", Int. J. Comput. Appl., vol. 119, no. 24, pp. 1, 2015.

3. V. Mulloni, B. Margesin, P. Farinelli, R. Marcelli, G. De Angelis, "Cycling reliability of RF-MEMS switches with gold-platinum multilayers as contact material", Proc. Symp. Design Test Integr. Packag. MEMS MOEMS, pp. 1-5, 2015.

4. G. M. Rebeiz, C. D. Patel, S. K. Han, C. H. Ko, K. Ho, "The search for a reliable MEMS switch?", IEEE Microw. Mag., vol. 14, no. 1, pp. 57-67, Jan./Feb. 2013. 
5. A. Basu, R. P. Hennessy, G. G. Adams, N. E. McGrue, "Hot switching damage mechanisms in MEMS contacts-Evidence and understanding", J. Micromech. Microeng., vol. 24, no. 10, pp. 105004, 2014.

6. R. Holm, Electric Contacts, New York, NY, USA:Springer-Verlag, pp. 1-55, 1967.

7. M. Braunovic, N. K. Myshkin, V. V. Konchits, Electrical Contacts: Fundamentals Applications and Technology, Boca Raton, FL, USA:CRC Press, 2006.

8. Z. Yang, D. Lichtenwalner, A. Morris, J. Krim, A. I. Kingon, "Contact degradation in hot/cold operation of direct contact micro-switches", J. Micromech. Microeng., vol. 20, no. 10, pp. 105028, 2010.

9. T. V. Laurvick, R. A. Coutu, "Experimental validation of external load effects on micro-contact performance and reliability", Proc. IEEE 61st Holm Conf. Electr. Contacts, pp. 353-357, Oct. 2015.

10. C. Stilson, R. Coutu, "Reliability evolution of Au-Au Au-Ru and Au-RuO 2 micro-contacts ", Proc. 27th Int. Conf. Electr. Contacts (ICEC), pp. 1-6, Jun. 2014.

11. C. Stilson, R. Coutu, "Contact resistance evolution of highly cycled lightly loaded micro-contacts", Proc. SPIE, vol. 8975, pp. 89750F, Mar. 2014.

12. G. M. Rebeiz, RF MEMS: Theory Design and Technology, Hoboken, NJ, USA:Wiley, 2003.

13. B. Toler, R. Coutu, "Characterizing external resistive inductive and capacitive loads for microswitches" in MEMS and Nanotechnology, New York, NY, USA:Springer, vol. 6, pp. 11-18, 2013.

14. T. Laurvick, C. Stilson, R. Coutu, "Experimental investigation of thin film spreading resistance in micro-contacts", Proc. IEEE 60th Holm Conf. Electr. Contacts (Holm), pp. 1-6, Oct. 2014.

15. T. V. Laurvick, R. A. Coutu, "Micro-contact performance and reliability under low frequency low amplitude alternating current (AC) test conditions", Proc. IEEE 61st Holm Conf. Electr. Contacts, pp. 222-226, Oct. 2015. 